models. Ultimately these studies may identify new therapeutic targets for CKD patients, to reduce their cardiovascular risk.

\section{IDENTIFYING A NOVEL ROLE FOR PMCA1 (ATP2B1) IN HEART RHYTHM INSTABILITY}

Claire Wilson*, Elizabeth Cartwright, Min Zi, Yatong Li. University of Manchester

10.1136/heartjnl-2017-311726.214

Arrhythmias continue to be a leading cause of death and disability across the world, and genetics are one of the mechanisms that are known to increase susceptibility. By identifying new genetic influences and further understanding the pathways involved in heart rhythm control we can begin to tackle some of the main challenges facing treatment development.

Here we aim to identify a new role for a gene linked to several features of heart failure Atp2b1 (Plasma membrane calcium ATPase 1, PMCA1). Along with its role in hypertension and other aspects of cardiac physiology, we believe PMCA1 may also influence heart rhythm stability and consequently the development of arrhythmias.

To investigate the role of PMCA1 in cardiac rhythm, cardiomyocyte-specific knockout mice (PMCA1 $1^{\mathrm{CKO} \tilde{A}, \hat{A}}$ ) were generated. In vivo electrocardiography showed PMCA1 ${ }^{\mathrm{CKO}}$ displayed signs of cardiac repolarisation dysfunction related to prolonged QT and JT intervals. Supplementary analysis using Langendorff-perfused hearts revealed PMCA1 ${ }^{\mathrm{CKO}}$ hearts have prolonged action potential duration compared to controls. Additionally using the methods highlighted above, PMCA1 ${ }^{\mathrm{CKO}}$ mice were shown to have an increase arrhythmia susceptibility to both in vivo and ex vivo programmed electrical stimulation. Further echocardiography and histological analysis showed these heart rhythm abnormalities occur in the absence of detectable structural heart disease with PMCA1 ${ }^{\mathrm{CKO}}$ cardiac structure and function being comparable to controls.

Our findings suggest a novel role for PMCA1 in heart rhythm stability, distinct from other cardiac disease. Furthermore, alterations in expression of Atp2b1 could influence an individuals susceptibility to developing arrhythmias.

\section{GENETIC ABLATION OF MICROTUBULE-ASSOCIATED PROTEIN 1S (MAP1S) PROTECTS THE HEART FROM PATHOLOGICAL HYPERTROPHY VIA REGULATION OF AUTOPHAGY}

Yulia Suciati Kohar*, Mohammed Najai, Nicholas Stafford, Min Zi, Sukhpal Prehar, Delvac Oceandy. University of Manchester

\subsection{6/heartjnl-2017-311726.215}

Background Autophagy is a process essential in maintaining cellular homeostasis, by degrading and recycling unwanted materials such as misfolded proteins and dysfunctional organelles. In the heart, autophagy is key in mediating pathological processes such as hypertrophy and remodelling. Autophagy is tightly regulated by a number of proteins and defective autophagy in response to pathological stimuli may lead to the development of adverse remodelling and eventually heart failure. In this study we investigated the role of microtubule-associated protein $1 \mathrm{~S}$ (MAP1S) in regulating autophagy during a number of cardiac pathological conditions. MAP1S has previously been identified as an interacting partner of the major autophagy regulator LC3; however, its role in the heart is unknown.

Results We used siRNA gene silencing to knockdown MAP1S in neonatal rat cardiomyocytes (NRCM) and detected the autophagic flux using GFP-LC3 expressing adenovirus. Following stimulation with rapamycin $(5 \mathrm{uM})$ and chloroquine (3 uM) for 2 hours, NRCM lacking MAP1S exhibited an increase in autophagy as indicated by a significant elevation in GFP-LC3 puncta formation. To confirm this finding we cultured fibroblasts from MAP1S knock out (MAP1S-/-) mice and induced autophagy using the same stimulus. Consistently, MAP1S-/- fibroblasts also showed increased autophagy after rapamycin/chloroquine treatment. Interestingly, the expression of autophagic modulators LC3II, Beclin and p62 did not differ between cells lacking MAP1S and controls, suggesting that MAP1S might affect autophagosome elongation and not the initiation process. In vivo, we confirmed higher autophagy in MAP1S-/- mice following rapamycin/chloroquine intraperitoneal injection as indicated by the number of amphisomes detected by electron microscopy. Next, to test the effects of pathological stimuli we subjected MAP1S-/- mice to transverse aortic constriction (TAC, 2 weeks) or myocardial infarction (MI, 4 weeks). Following TAC, MAP1S-/- mice displayed less hypertrophy as indicated by heart weight/body weight ratio, cardiomyocyte surface area (histology) and the expression of hypertrophic markers. Consistently, after MI MAP1S-/- mice also showed a reduction in hypertrophy.

Conclusions Our findings suggest that genetic inhibition of MAP1S induces autophagy in cardiomyocytes, whereas in vivo ablation of this gene in mice reduces cardiac hypertrophy in response to pathological stimuli such as TAC and MI.

\section{KNOCKOUT P47PHOX REDUCES ANGIOTENSIN II- INDUCED CARDIAC OXIDATIVE STRESS AND HYPERTROPHY}

Fangfei Liu*, Junjie Du, Jian-Mei Li. School of Biological Sciences, University of Reading

\subsection{6/heartjil-2017-311726.216}

Oxidative stress due to the activation of a Nox2-containing NADPH oxidase is involved in Angiotensin II (AngII)-induced cardiovascular dysfunction. $\mathrm{p} 47^{\mathrm{phox}}$ is a key regulatory subunit of Nox2. However, the role of $\mathrm{p} 47^{\text {phox }}$ in AngII-induced cardiac damage remains unclear. In this study, we used littermates of C57BL/6 wild-type (WT) and $\mathrm{p} 47^{\text {phox }}$ knockout $(\mathrm{KO})$ mice $(n=7)$ at the age of $10 \sim 12$ months to investigate the effect of p47 $7^{\text {phox }}$ knockout on AngII-induced cardiac oxidative stress and hypertrophy. In WT mice, AngII infusion $(1 \mathrm{mg} / \mathrm{kg} / \mathrm{day}$ for 14 days) increased significantly the systolic blood pressure (SBP) from $127 \pm 13$ to $172 \pm 11 \mathrm{mmHg}$, and this was accompanied with significant indication of cardiac hypertrophy (heart/body weight ratio increased $\sim 17.9 \pm 0.1 \%$ ) as compared to vehicle infused controls $(\mathrm{p}<0.05)$. However, in $\mathrm{p} 47^{\text {phox }} \mathrm{KO}$ mice, AngII infusion caused a mild increase in SBP (from 119 \pm 9 to $149 \pm 10 \mathrm{mmHg}, \mathrm{p}<0.05$ ) without significant increase in heart/body weight ratio. Cardiac production of reactive oxygen species (ROS) was examined by both lucigenin-chemiluminescence and DHE fluorescence. Compared to vehicle controls, 